\title{
Depth encoded input polarisation independent swept source cross-polarised optical coherence tomography probe
}

\author{
Katharina Blessing ${ }^{1,2, *} \mathbb{D}$, Judith Schirmer ${ }^{1,2}$, Asha Parmar ${ }^{1,2}$ (ib) and Kanwarpal Singh Ka $^{1,2}$ \\ ${ }^{1}$ Max-Planck Institute for the Science of Light, Research Group Singh, Staudtstraße 02, 91058 Erlangen, \\ Germany \\ ${ }^{2}$ Friedrich-Alexander Universität Erlangen-Nürnberg, Department of Physics, Staudtstraße 07, 91058 \\ Erlangen, Germany \\ E-mail: Katharina.Blessing@mpl.mpg.de
}

Received 14 December 2020, revised 25 March 2021

Accepted for publication 28 April 2021

Published 17 May 2021

\begin{abstract}
Within the last decades, several studies have been published that prove the benefit of polarisation sensitive optical coherence (psOCT) tomography for the field of biomedical diagnostics. However, polarisation sensitive imaging typically requires careful control of the polarisation state of the input illumination, which leads to bulky and delicate systems. While psOCT provides quantitative information, it is mostly sufficient to analyse the images qualitatively in the field of biomedical diagnostics. Therefore, a reduced form of this technique, cross-polarised optical coherence tomography (cpOCT), moves into the focus of interest that serves to visualise the birefringence properties of a sample. Despite the low requirements for the illumination's polarisation, most of the proposed systems still include complex illumination control mechanisms. Here, we propose a common path probe based endoscopic system with an lateral resolution of $30 \mu \mathrm{m}$ and a sensitivity of $103 \mathrm{~dB}$ comprising a commercially available swept-source OCT system and a free-space module which does not require any polarisation controlling elements. A Faraday mirror substitutes the complex polarisation control apparatus. We demonstrate the independence of the approach from the polarisation state of the light source by monitoring the illumination power in the orthogonal channels while varying the source polarisation. Furthermore, we validate the ability of the system to reveal the birefringence properties of different samples, starting from a quarter-wave plate, since its properties are fully characterised. Additionally, we present imaging results from several tissues to demonstrate its feasibility for the field of biomedical diagnostics.
\end{abstract}

\footnotetext{
Author to whom any correspondence should be addressed.
} 
Supplementary material for this article is available online

Keywords: Optical coherence tomograph, interference methods, endoscopy, optical biopsy, polarisation sensitive methods, medical imaging

(Some figures may appear in colour only in the online journal)

\section{Introduction}

Since its development in 1991 [1], optical coherence tomography (OCT) found multiple applications in the field of biomedical diagnostics. As the optical equivalent to ultrasound imaging, OCT opens the possibility to visualise tissue structures up to an imaging depth of $3 \mathrm{~mm}$ with sub-micron to few tens of microns axial and lateral resolution. Thus, it provides a depth encoded cross-sectional image of the sample, whereas conventional microscopes picture the surface structure of it. Further, a three-dimensional representation of the sample is feasible. In the field of biomedical imaging, this gives the option to detect malignant tissue areas before they become prominent on the surface.

After proving its utility in ophthalmology [2, 3] where it became a standard diagnosis instrument [4], it is used in the gastrointestinal tract [5, 6], to detect pulmonary lesions [7], to image coronary arteries [8], to map lymph nodes in case of breast cancer [9], and for diagnostics in the field of oncourology [10]. The improvement of OCT technologies has been summarised in several reviews within the last years [11-14].

Another class of OCT, polarisation sensitive OCT (psOCT), reveals the phase changes the illumination undergoes while traveling through the sample. psOCT gives access to the full set of Stokes vectors, and reveals the entire polarisation state of the light coming from the sample under the condition that the polarisation state of the sample illumination is fully controlled. Advancements in this field and its benefits for the field of biomedical imaging were recently discussed by de Boer et al [15] and by Baumann [16].

However, full control of the sample illumination mostly leads to bulky systems due to several phase controlling elements [17]. In biomedical diagnosis, it is oftentimes sufficient if the images show phase induced effects qualitatively, in particular the birefringence properties of the sample. Therefore, another class of psOCT devices called cross-polarised OCT (cpOCT) gained importance. These systems require to image the sample in two orthogonal channels, one with the same polarisation as the illumination and another with a polarisation orthogonal to it [18]. Aiming flexible endoscopic systems, where at least the orientation to the optical axis changes randomly because of bending and stretching of the probe, the lowered requirements for the polarisation control lead to the option of developing compact approaches [19].

Several clinical studies show the relevance of these endoscopic devices in the field of medical diagnosis, since especially fibrous tissue in healthy state shows different birefringence properties compared to diseased tissue. In fact, a swept-source based OCT system containing a rigid probe is commercially available [20]. For brain imaging with cpOCT, assessment criteria to distinguish between tumorous and nontumorous tissue were defined [21]. Furthermore, in cardiology, it helps to distinguish fibrotic myocardium from normal or ablated one [22, 23].

As mentioned before, OCT is the optical equivalent to ultrasound imaging, i.e. it detects the echo of a signal originating from a light source and reflecting from a sample. Compared to ultrasound imaging which detects it directly, OCT detects the interference of the scattered signal with a reference signal. The core part of firstly reported approaches is a Michelson interferometer. OCT signal, in this case, consists of a line, so-called A-scans, which contain the interference signal created by the interferometer while scanning a mirror physically over a defined distance [1]. Thus, imaging speed is limited by the distance the mirror travels within one scan. Many applications, in particular in-vivo, require video or at least near-video imaging rates. To overcome the limitations in the acquisition speed, another type of OCT systems was suggested. Instead of detecting the interference signal in real-space, these systems work in the frequency domain [24]. The interference pattern is acquired either by using a spectrometer with a particular wavelength spreading mechanism or by sweeping sequentially through different wavelengths, applying a swept-source with known sweeping rate and wavelength range (ssOCT). In clinical praxis, spectrometer based systems became state-of-theart [17], though swept-source based systems even enhance the scanning speed [25]. Experimentally, a source with a scanning speed of $28 \mathrm{MHz}$ has been reported in 2015, developed to perform quantitative phase imaging [26].

Further advantages of ssOCT compared to spectrometer based systems are a higher spectral resolution due to the narrow line-width of the swept-source, a higher sensitivity, and an extension of the imaging depth. In many cases, noise can be further reduced by balanced detection where the common noise in reference and sample arm cancels out. The improvements balanced detection adds to the image quality have been briefly discussed by Podoleanu [27].

However, most of the clinical systems used for imaging the internal organs are fibre based which suffer from random polarisation changes and is sensitive to tiniest movements of system fibres. Thus, to detect phase shifts of the sample illumination, the system has to be stationary, and the polarisation state has to be controlled carefully during the measurement. For systems applied under laboratory conditions, this affects minimally, but in the clinical setting, a robust system is required. We show that our approach despite its simplicity 
is insensitive to changes in the polarisation during the measurement.

For optical fibre based systems, even if the sample polarisation measurement is not of interest, reference and sample arm of the system still have to be carefully matched for dispersion and phase [28]. Otherwise the imbalances within both arms introduce imaging artifacts. In particular dispersion imbalances occur frequently. Therefore, several approaches were demonstrated to face the problem [29]. Specifically in endoscopic approaches with elastic probes, where bending and stretching of it during the measurement causes systematically induced changes in length and polarisation of the sample arm, to balance both arms is one of the main challenges in the development. The evolvement of endoscopic systems was summarised by Gora et al [30].

One simple and effective method to circumvent the formation of dispersion imbalances and length mismatches between reference and sample signal is to build common-path probes (CPPs) [31]. Within these probes, reference and sample arm follow the same fibre. Interference takes place on a reflecting surface at the tip of the probe. Thus with fibre manipulation, reference and sample signals are affected similarly. In simplest CPP, the reflecting surface on tip of a bare single-mode fibre (SMF) serves as interference plane [32]. However, these probes come with a fixed working distance and reference illumination power. In the system reported here, we used a customised monolithic mirror based CPP. The previously reported design opens the possibility to adjust the reference power independently from the working distance [33].

Though light travelling through SMF does not maintain its polarisation state while bending and stretching, the orthogonality of the beams is maintained over the entire distance. Gelikonov et al made use of this property to design a CPP based cpOCT system in 2006 [19]. In 2018, they reported an improved spectrometer based system [34] which relies on the active maintenance of the circular polarisation during the measurement.

CPP based cpOCT systems require the sample to be illuminated with two orthogonal signals of equal intensities. Recently, we demonstrated a CPP based cpOCT system using a depolariser. Due to the depolariser, the beams are more or less equal in power. However, the system still shows some fluctuations in the sample power during the measurement [35]. Another option to create an input-polarisation independent approach is to implement a Faraday mirror which introduces a delay of $90^{\circ}$ between the vertical components of the input light making it orthogonal. A spectrometer based approach of this kind applying a superluminescent light source was introduced in 2015 by Gurbakova et al to characterise atherosclerotic plaques [22] where co-polarised and crosspolarised images were obtained sequentially. We display an even simpler system combining the free space module with the commercially available ssOCT engine and the monolithic CPP such that the co-polarised and the cross-polarised images are acquired simultaneously using a depth encoded scheme.

\section{System description}

Figure 1 illustrates the system schematically. The OCT engine which is commercially available at Axsun technologies (North Billerca, Massachusetts) comprises the illumination and the data acquisition module. The illumination module basically provides a swept source with a central wavelength of $1310 \mathrm{~nm}$, a scan range of $140 \mathrm{~nm}$ and a sweeping rate of $100 \mathrm{kHz}$. The source comes with a maximal output power of $24 \mathrm{~mW}$. On the other hand, the data acquisition module consists of a detection unit containing two photodiodes which enables the system to perform balanced detection. As we are demonstrating an approach applying a CPP, we use just one of the diodes running the system in unbalanced configuration. This diode is connected to a data acquisition (DAQ) card, a digitiser, and a field programmable gate array. The full data processing is performed within this module such as the engine gives the depth encoded sample images as an output which depict the intensities.

To perform cpOCT, we connected the engine to a free space module, and to collimate the illumination within the module, as well as for signal collection, the system contains two fibre collimators delivered by Oz optics (HPUCO-13A-1300/1550s-6.2AS, AMS Technologies AG), one at the illumination output and one connected to a faraday rotator mirror with bulkhead connector from Newport (F-FRM-1-NT-FA, Newport Corporation, California). A non-polarising beam splitter (BS012, Thorlabs, Inc.) (BS) divides the signal from the laser into two beams of equal power. One of the beams is reflected by the Faraday mirror whereas the other is reflected by a silver mirror positioned on a translational stage. The polarisation of the illumination returning after reflection from the Faraday mirror is rotated by an angle of $90^{\circ}$ whereas the polarisation state of the illumination is maintained during the reflection from the silver mirror. The reflected signals are recombined again by the BS into one beam which contains two orthogonal signals separated by the optical path difference between the silver and the Faraday mirror. For cpOCT, the optical path difference between the orthogonal channels (co- and crosschannel) has to fit perfectly to get a depth encoded image comprising both signals. Therefore, the silver mirror is mounted on a translational stage to allow fine adjustment of the optical path difference (OPD).

To couple the recombined beam into the probe and to avoid a back-coupling into the laser, a circulator (CIR-1310-50APC, Thorlabs Inc.) is positioned between the light source and the input-collimator to the free space system. Considering that the circulator contains only one more free port but two are required, one for the connection to the CPP and one to connect the system to the data acquisition module, a second circulator is connected to the third port of the first one. This way the signal is transported to the CPP and from the CPP to the photodiode, as depicted schematically in figure 1. For the proof of principle, the forward viewing probe is connected to an electronic scanning device and positioned above the sample. This way, flat samples are scanned laterally. 


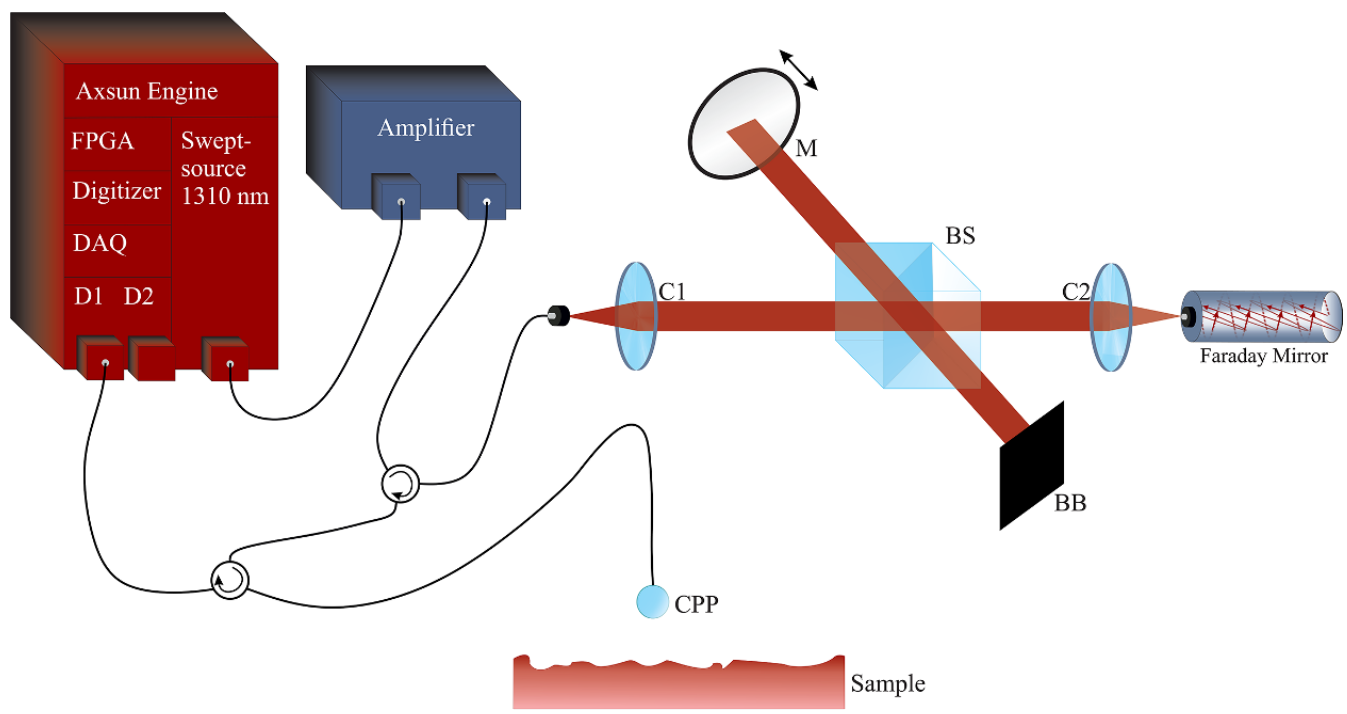

Figure 1. Scheme of the cross-polarised OCT system. The Axsun engine with swept-source, photodiodes (D1, D2) and data acquisition module containing a DAQ-card a field programmable gate array and a digitiser is connected to an amplifier. Besides the faraday mirror the free space module contains two collimator $(\mathrm{C} 1, \mathrm{C} 2)$, a non-polarising beam splitter (BS), mirror (M) on a translational stage and a beam blocker (BB). Via circulators the system is connected to the engine and the common-path probe (CPP).

To build the CPP, we attached a spacer of 250 microns to an SMF and coated the tip with a $7 \mathrm{~nm}$ thick gold layer before we attached a $500 \mu \mathrm{m}$ ball lens to it. Interference takes place at the mirror surface. The gold layer reflects approximately $50 \%$ of the light and transmits the rest. A fraction of the reflected light is coupled back to the core of the fiber and acts as reference signal. In our study, we fabricated a probe that provided $50.6 \mu \mathrm{W}$ reference power in combination with the laser and the amplifier [31].

A major disadvantage of the system is the significant losses resulting from different components, namely the coupling losses at the Faraday mirror, and the circulators themselves which introduce a loss of about $30 \%$ each. All these losses sum up. Additionally, the semi-transparent mirror at the tip of the CPP halves the remaining signal before hitting the sample. This results into a sample illumination which is insufficient for tissue imaging. To compensate for this, we enhance the input illumination power with an amplifier (Series CLD1000, Model CLD1015, Thorlabs Inc. BOA1130S, Thorlabs Inc.).

CpOCT requires two images of the same sample area. Therefore, the polarisation of the illumination used to acquire the first image has to be strictly orthogonal to the second one [36]. Alternatively both images can be taken simultaneously [17], like in our case, applying light which contains both polarisation states at once. Resulting from different OPD of the two polarisation states between the reference signal reflected from the reference mirror and the sample signal reflecting from the sample, the signal separates into three different depth encoded images of the same sample region. The initial OPD between the two orthogonal states is generated by the distance between the Faraday mirror and the silver mirror $\Delta d_{1}$. In case of CPP based systems, if the distance between the sample and the reference mirror is $\Delta d_{2}$, one image arises at $\Delta d_{2}$, in the following called co-image, and another two images arise at the positions $\Delta d_{2}-\Delta d_{1}$ and $\Delta d_{2}+\Delta d_{1}$. The cross-signal results out of a combination of the latter two images. Thus, the intensity of the cross-image $I_{\text {cross }}$ is computed out of the intensity of the upper image $I_{\text {cross1 } 1}$ and the intensity of the lower image $I_{\text {cross2 }}$ by equation (1).

$$
I_{\text {cross }}=\sqrt{I_{\text {cross } 1}^{2}+I_{\text {cross } 2}^{2}}
$$

Out of the intensity distributions of the co- $I_{\mathrm{co}}$ and the crossimage $I_{\text {cross }}$, the reflectivity $R$ of the sample is evaluated by equation (2)

$$
R=\sqrt{I_{\mathrm{cross}}^{2}+I_{\mathrm{co}}^{2}}
$$

To visualise the differences between co- and cross-signal, i.e. to elucidate the birefringence properties of the sample, we calculate the depolarisation ratio $\delta$ out of $I_{\text {cross }}$ and $I_{\text {co }}$ like suggested by Schmitt and Xiang [18] using equation (3). While in psOCT it is possible to calculate sample's retardance directly, cpOCT provides an effective retardance [37] which is given by the arcus-tangent of equation (3). The effective retardance can be and has been previously used to increase the tissue contrast in brain tumor [21] and cardiac tissue imaging [22, 23].

$$
\delta=\frac{I_{\text {cross }}}{I_{\mathrm{co}}}
$$

We analysed the data extracting the processed images from the engine and we separated the areas containing the co-from the two cross-images. Then, we determined the cross-image by applying equation (1) pixelwise to the images. Further processing steps include generating the regular OCT image applying equation (2) also to each pixel, and determining the depolarisation ratio using (3). Of the datasets from different tissue we extract the co-image, the cross-image, the regular image, and the depolarisaiton ratio $\delta$. 

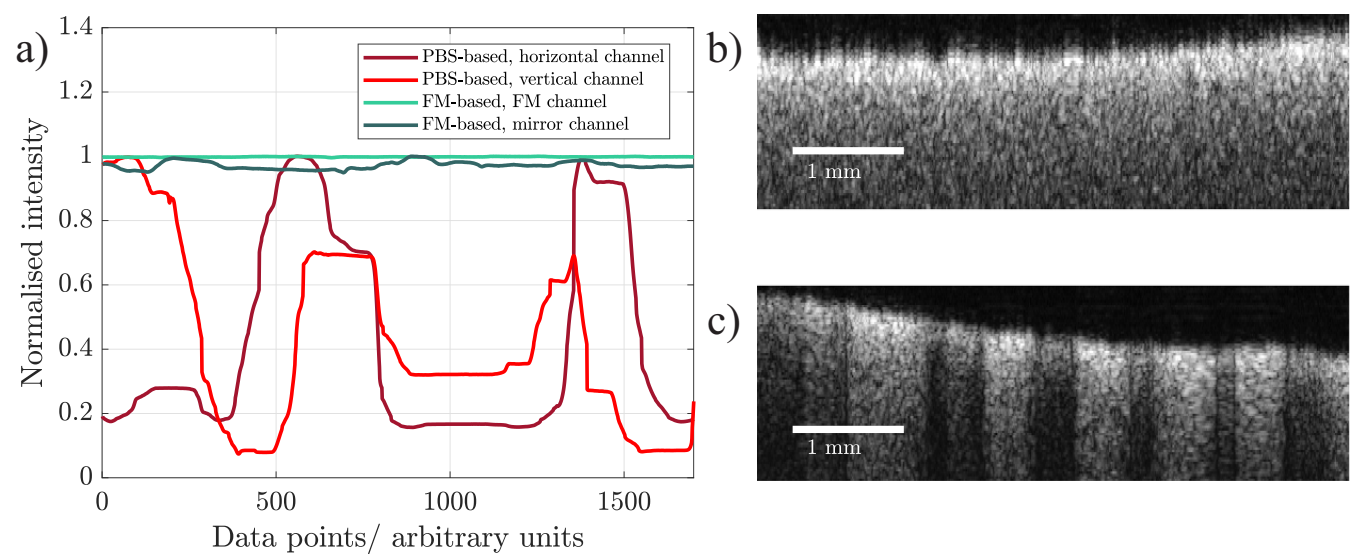

c)

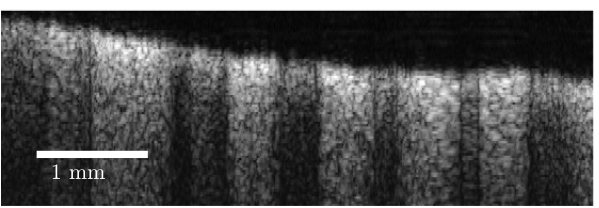

Figure 2. Fluctuations of the sample illumination. (a) The graph shows the fluctuations of the sample illumination power in the orthogonal channels normalised to the maximal power achieved. The results acquired with the system reported here (FM-based) are shown in green, while the acquisition results from the system used for the comparison (PBS-based) are pictured in red (one line for each orthogonal channel, two channels per system). (b) Cross-signal of a paper scanned with the FM-based approach. (c) Cross-signal of a paper scanned with the PBS-based approach.

We determined the lateral resolution of the system by scanning a variable line grating from Thorlabs (R1L3S6P, Thorlabs Inc.), and to measure the sensitivity, we made use of a method described by Singh et al [38]. Due to the short working distance, it is difficult to apply the common method of sensitivity measurement, i.e. by introducing an attenuator into the optical pathway. Therefore, instead of an attenuator in the optical pathway, first, we maximised the light coupling into the CPP from a mirror and then we misaligned the sample mirror to reduce the back coupling of the signal by a known value. For practical reasons, we chose a factor $10^{-2}$ of the initial value, i.e. a sensitivity reduction of $20 \mathrm{~dB}$. Afterward, we measured the signal to noise ratio (SNR) of the mirror A-scan and added the reduction to obtain the system's sensitivity.

We monitored the fluctuations in the sample illumination after blocking one of the orthogonal channels while changing the polarisation of the input-illumination. Depolarisation in the illumination signal from a quarter-wave plate imaged at different illumination angles was measured. We computed also the Michelson-contrast between co- and cross-signal for paper as well as for mirrors. Last, imaging data of different kinds of tissue are presented to motivate the applicability in the field of biomedical diagnostics.

\section{System performance}

The system as described above comes with an axial resolution of $6 \mu \mathrm{m}$, a lateral resolution of $30 \mu \mathrm{m}$, a sensitivity of $103 \mathrm{~dB}$ with $1 \mathrm{~dB}$ sensitivity roll-off for the full imaging range. Unlike spectrometer based systems which suffer from significant sensitivity roll-off and require sensitivity adjustments in the images, our system does not require such adjustments. The sensitivity is approximately the same throughout the imaging range. We have used an amplifier in our system which also amplifies the system's noise; however, the increase in the signal is higher than the noise. We measured the SNR of the system with and without amplifier and obtained an increase of $5.5 \mathrm{~dB}$ in the SNR. The stability measurements, see figure 2, reveal the importance of a stable illumination power during the measurement. To monitor the effect of input polarisation changes on the sample illumination, we positioned a manual two paddle polarisation controller (FPC020, Thorlabs Inc.) at the input-illumination fibre. For the measurements, we blocked the orthogonal channels subsequently taking measurements for each. In this configuration, we monitored the fluctuation in the sample power with a powermeter (PM400, Thorlabs, Inc.) while moving the paddles of the polarisation controller. To compare the performance of our current approach with the previously described methods [35] which used a polarising beam splitter (PBS) to obtain two orthogonal illumination signals, we compared the fluctuation in the sample illumination intensity while changing the source polarisation. Instead of the Faraday mirror the other system contained a simple mirror, whereas a PBS was placed instead of the non-polarising one (BS). A schematic of the alternative system can be found in the supplemental material (available online at stacks.iop.org/JPD/54/305401/mmedia), see supplemental figure S1. For a better comparison of the data, we normalised the intensity measurements to the maximally achieved power. The graph, figure $2(a)$, demonstrates a fluctuation of $5.1 \%$ in the first channel and a fluctuation of $0.5 \%$ in the second channel of the approach presented in this paper, i.e. the system provides a stable illumination in the orthogonal channels.

To emphasise the importance of a constant sample illumination we imaged paper, a highly scattering material which ideally contains a homogeneous cross-signal while moving the paddles of the polarisation controller during the image acquisition. In figure 2(b) the dataset from the Faraday mirror based system is displayed whereas (c) shows the image taken with the simple approach. Instead of a homogeneous intensity distribution picture (c) depicts horizontal stripes. However, the intensity distribution in (b) is homogeneous which demonstrate that the source polarisaiton has minimal effect on the image quality. 

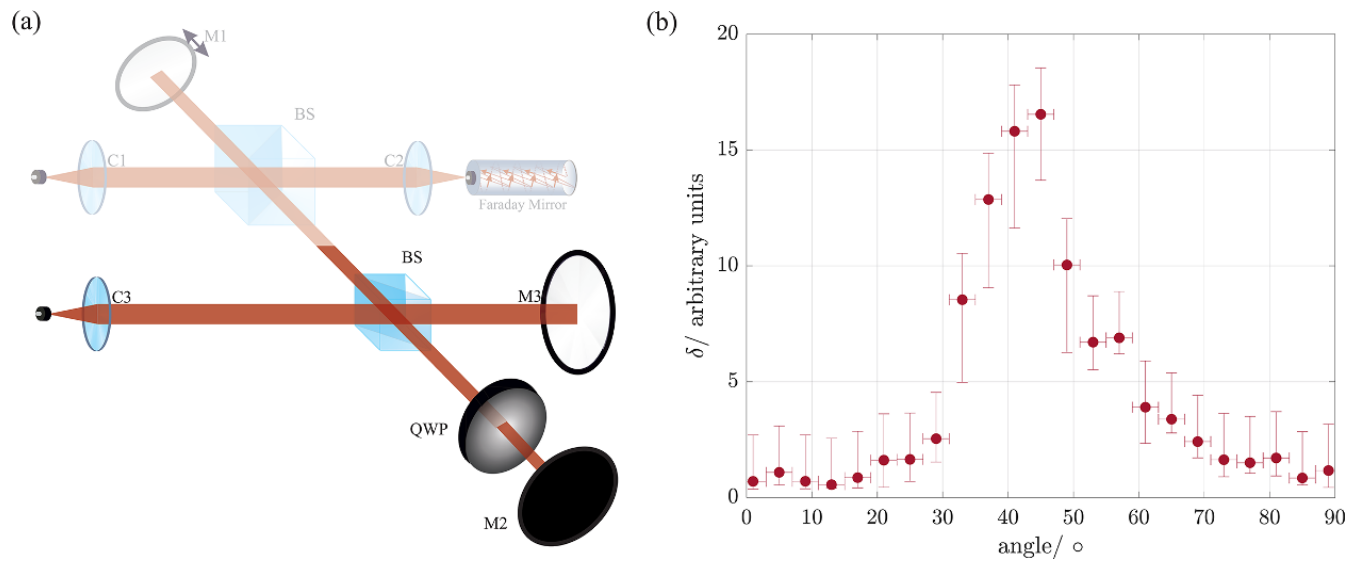

Figure 3. Angular dependency of the depolarisation ratio of a quarter-wave plate. (a) Schematic representation of the system modifications. (b) Graph of the angular dependency of the depolarisation ratio.

(a)

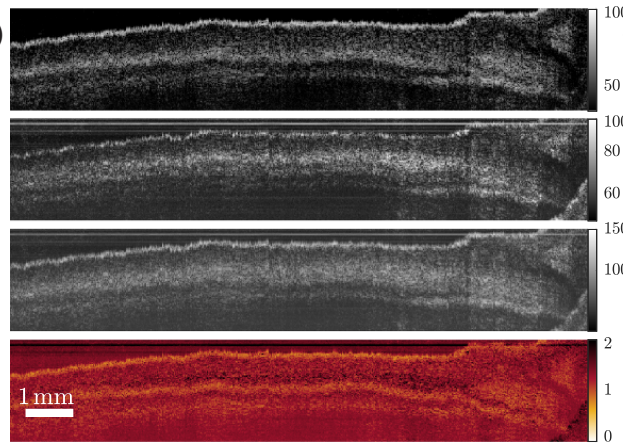

(b)

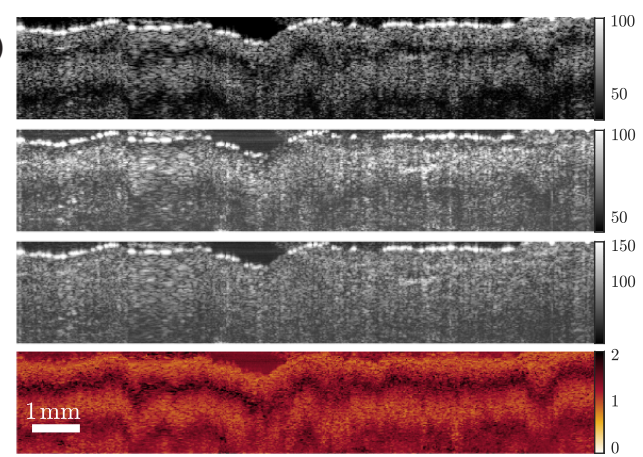

Figure 4. Tissue imaging results. Datasets of tissue images comprising four images each, from top to bottom: the cross-signal, the co-signal, the reflectivity and the depolarisation ratio $\delta$. (a) Nailbed of a human volunteer. (b) Cow muscle tissue.

In a next step, we determined the ability of the system to retrieve the birefringence properties of the sample. Therefore, we acquired the signal reflected from a mirror after passing a quarter-wave plate. Since the working distance of our probe of approximately $3 \mathrm{~mm}$ is too short to position a quarterwave plate in the beam-path, for this experiment we had to slightly modify the system as illustrated in figure 3(a). Basically, we transferred the system into a bench-top approach. Thus, instead of the probe we extended the free-space module with another non-polarising beam splitter to split the illumination into sample and reference arm of the same length and put mirrors at both ends. The quarter-wave plate was placed in one of the arms which was dedicated to be the sample arm. Furthermore, another collimator was required to collect the signal and lead it to the detector. The quarter-wave plate was mounted in a rotational mount. We acquired one image every four degrees and determined the depolarisation ratio of each averaging the A-scans. Subsequently, we plotted these values with respect to its relative angle. The graph demonstrates the expected behaviour for this kind of birefringent material.

Another value for the effectivity of the system is the modulation of co- and cross-image in case of a non-birefringent material, like a mirror, and a highly scattering material, like paper. In the first case, the cross-signal should vanish entirely whereas, in the second case, the signal should be equally distributed between co- and cross-signal. As a quantitative measure we decided to compute the Michelson contrast as described in equation (4) for both cases whereas in the ideal case the contrast should vanish for the paper signal, and maximise for the mirror signal.

$$
C=\frac{\left|I_{\mathrm{co}}-I_{\text {cross }}\right|}{I_{\mathrm{co}}+I_{\text {cross }}} \quad 0 \leqslant C \leqslant 1 .
$$

Imaging the paper results in a contrast of $0.10 \pm 0.03$ and of the mirror in $0.86 \pm 0.04$. Contemplating the fact that we did not correct for background noise these values comes close to the expected values. That in turn indicates, that the Faraday mirror, though optimised for a wavelength of $(1310 \pm 15) \mathrm{nm}$ with an operation bandwidth of $15 \mathrm{~nm}$, does not introduce significant inhomogeneities which decrease the modulation efficiency over the entire spectral range of the swept source.

As mentioned before, various tissue contain characteristic birefringence properties. To elucidate the ability of the approach to reveal these properties we imaged different kinds of tissue. Firstly, we acquired data from the nailbed of a human volunteer because of its characteristic laminar structure, which was presented exemplarily in earlier publications [17, 39]. Additionally, we imaged cow-muscle tissue provided by a local butcher shop. The maximal imaging depth of OCT at $1310 \mathrm{~nm}$ in cow-muscle tissue is approximately $3 \mathrm{~mm}$. However, the depth range of the engine is limited to $5 \mathrm{~mm}$ and 
and about $1 \mathrm{~mm}$ is lost by some interference signal emerging from different reflective surfaces within the system. Since all three images have to appear within the leftover $4 \mathrm{~mm}$ and their signal should not overlap, we prepared thin samples of the muscle tissue of approximately $0.5 \mathrm{~mm}$. One exemplary imaging result from both kinds of tissue is represented in figure 4. In both cases, the images display laminar structures of alternating intensity caused by the birefringence properties of the tissue. These structures smear out, see figure 4, respectively vanish in the regular OCT image which points out that cpOCT imaging retrieves relevant additional information about the tissue structure compared to simple OCT approaches.

In birefringent material, the co-signal minimises when the cross-signal maximises and vice versa. This behaviour is more evident, especially in the data-set of the cow-muscle tissue, figure 4(b). It is also worth mentioning that the image quality is achieved without any post-processing.

\section{Conclusion}

In summary, we present a robust cpOCT approach. Though it is an endoscopic system, it provides with an lateral resolution of $30 \mu \mathrm{m}$ and a sensitivity of $103 \mathrm{~dB}$ a performance comparable to previously reported bench-top systems [17]. Contrary to the simple system containing a two paddle-polarisation controller and a polarising beam splitter to realise the orthogonal polarisation states, it is almost entirely independent of the polarisation state of the light source, see figure 2. In the clinical setting, especially during surgery, robust, userfriendly, and portable machines are desirable. System transport causes vibrations of the illumination fibres. These vibrations might cause changes in the polarisation state of the output-beam. Under laboratory conditions, the alignment of the system can be adjusted unproblematically by a polarisation controller before the measurement. In the clinical setting, it might be required to change the position of the system during the measurement procedure. The stability of this approach allows a flexible repositioning within the procedure. Thus, it addresses in particular the clinical requirements.

As mentioned above, we applied a customised common path probe. This allows constant imaging results independently of bending and stretching of the probe since reference and sample arm follow the same path and undergo the same polarisation changes during the measurements. But, applying a common path probe comes with the disadvantage that balanced detection is not feasible any more which is commonly used in swept-source OCT systems to minimise the random intensity noise (RIN) from the laser. Consequently, it is not possible to remove the effect of RIN in our system which degrades the image quality.

Nevertheless, the characterisation measurements with a quarter-wave plate indicate that the system reliably displays the birefringence properties of the sample. As the modulation ranges within the expected values for unimpeded measurements, the frequency band of the light source seems not to disturb the measurement even though the Faraday mirror is designed for $1310 \mathrm{~nm}$ with a narrow tolerance.
In comparison to previously reported endoscopic crosspolarised systems, our current approach provides both co- and cross-signal in one shot while being independent of the light source polarisation. Thus, it provides a complete dataset of the tissue, which significantly increases the value of the provided information compared to systems which provide the co- and the cross-signal sequentially.

However, for a valid imaging result, the tissue has to be cut into thin slices as the imaging depth of this system is limited. We take the co- and cross-signal simultaneously. Within the image, the intensity values appear at certain positions encoded by the optical path difference. This difference can be adjusted by moving a mirror on a translational stage. For the image separation, it is necessary that the signals do not overlap, and thus an enhancement of the depth range by about a factor of two is required.

For a transfer from bench to bedside, besides the depth range, the design of the probe should be changed into a sideviewing one if one aims to image the luminar structures. An option to achieve that would be to polish the ball-lens tip such way that by total internal reflection the beam is led to the side of the probe instead of the front. The entire probe would be rotated by a motor inside a bio-compatible shielding tube. Depending on the application, a different design might be needed. Nevertheless, we expect that the concept presented here after insetting the suggestions above could lead to a powerful clinical diagnostic tool. Especially in the fields of coronary imaging, and brain tumor imaging, it will facilitate intraoperative diagnostics.

\section{Data availability statement}

The data that support the findings of this study are available upon reasonable request from the authors.

\section{ORCID iDs}

Katharina Blessing (D) https://orcid.org/0000-0002-23953440

Asha Parmar (D) https://orcid.org/0000-0002-5630-9483

Kanwarpal Singh (D) https://orcid.org/0000-0002-6884-8089

\section{References}

[1] Huang D et al 1991 Science 254 1178-81

[2] Koo D, Park H C, Gehlbach P L and Song C 2016 Biomed. Opt. Express 7 4816-26

[3] Fercher A F, Hitzenberger C K, Kamp G and El-Zaiat S Y 1995 Opt. Commun. $11743-48$

[4] Monroy G L, Won J, Jr D R S, Dsouza R and Boppart S A 2017 J. Biomed. Opt. 22121715

[5] Bouma B E, Tearney G J, Compton C C and Nishioka N S 2000 Gastrointest. Endosc. 51 467-74

[6] Sergeev A M et al 1997 Opt. Express 1 432-40

[7] Tsuboi M, Hayashi A, Ikeda N, Honda H, Kato Y, Ichinose S and Kato H 2005 Lung Cancer 49 387-94

[8] Bezerra H G, Costa M A, Guagliumi G, Rollins A M and Simon D I 2009 JACC: Cardiovascular Interventions 2 1035-46 
[9] Nguyen F T et al 2010 IEEE Eng. Med. Biol. Mag. 29 63-70

[10] Kharchenko S, Adamowicz J, Wojtkowski M and Drewa T 2013 Cent. Eur. J. Urol. 66 136-41

[11] Adhi M and Duker J S 2013 Curr. Opin. Ophthalmol. 24 213-21

[12] Fercher A F, Drexler W, Hitzenberger C K and Lasser T 2003 Rep. Prog. Phys. 66 239-303

[13] Shu X, Beckmann L J and Zhang H F 2017 J. Biomed. Opt. 22121707

[14] Zysk A M, Nguyen F T, Oldenburg A L, Marks D L and Md S A B 2007 J. Biomed. Opt. 12051403

[15] de Boer J F, Hitzenberger C K and Yasuno Y 2017 Biomed. Opt. Express 8 1838-73

[16] Baumann B 2017 Appl. Sci. 7474

[17] Sharma G, Sharma S, Blessing K, Hartl G, Waldner M and Singh K 2020 J. Opt. 22045301

[18] Schmitt J M and Xiang S H 1998 Opt. Lett. 23 1060-2

[19] Gelikonov V M and Gelikonov G V 2006 Laser Phys. Lett. 3445

[20] Lenton P, Rudney J D, Fok A and Jones R S 2014 J. Med. Imaging 1016001

[21] Yashin K S et al 2019 Front. Oncol. 9201

[22] Gubarkova E V et al 2016 J. Biophoton. 9 1009-20

[23] Yao X, Gan Y, Ling Y, Marboe C C and Hendon C P 2018 J. Biophoton. 11 e201700204

[24] Leitgeb R, Hitzenberger C K and Fercher A F 2003 Opt. Express 11 889-94

[25] Drexler W, Liu M, Kumar A, Kamali T, Unterhuber A and Leitgeb R A 2014 J. Biomed. Opt. 19071412
[26] Wei X, Lau A K S, Xu Y, Tsia K K and Wong K K Y 2015 Biomed. Opt. Express 6 3855-64

[27] Podoleanu A G 2000 Appl. Opt. 39 173-82

[28] Singh K, Sharma G and Tearney G J 2018 J. Opt. 20025301

[29] Wojtkowski M, Srinivasan V J, Ko T H, Fujimoto J G, Kowalczyk A and Duker J S 2004 Opt. Express 12 2404-22

[30] Gora M J, Suter M J, Tearney G J and Li X 2017 Biomed. Opt. Express 8 2405-44

[31] Singh K, Reddy R, Sharma G, Verma Y, Gardecki J A and Tearney G 2018 Lasers Surg. Med. 50 230-5

[32] Sharma U and Kang J U 2007 Rev. Sci. Instrum. 78113102

[33] Blessing K, Sharma S, Gumann A and Singh K 2019 Eng. Res. Express 1025008

[34] Gelikonov V M, Romashov V N, Shabanov D V, Ksenofontov S Y, Terpelov D A, Shilyagin P A, Gelikonov G V and Vitkin I A 2018 Radiophys. Quantum Electron. 60 897-911

[35] Blessing K, Schirmer J, Sharma G and Singh K $2020 \mathrm{~J}$. Biophoton. 13 e202000134

[36] Kuranov R V, Sapozhnikova V V, Turchin I V, Zagainova E V, Gelikonov V M, Kamensky V A, Snopova L B and Prodanetz N N 2002 Opt. Express $10707-13$

[37] Gubarkova E V et al 2016 J. Biomed. Opt. 21126010

[38] Singh K, Yamada D and Tearney G 2015 Sovrem. Tehnologii Med. 7 29-33

[39] Sharma S, Hartl G, Naveed S K, Blessing K, Sharma G and Singh K 2020 Rev. Sci. Instrum. 91043706 